\title{
Network Neutrality Debate and ISP Inter-Relations: Traffic Exchange, Revenue Sharing, and Disconnection Threat
}

\author{
Pierre Coucheney, Patrick Maillé, Bruno Tuffin
}

\begin{abstract}
The network neutrality debate originally stems from the growing traffic asymmetry between ISPs, questioning the established peering or transit agreements. That tendency is due to popular content providers connected to the network through a single ISP, and whose traffic is not charged by distant ISPs. We propose in this paper to review the economic transit agreements between ISPs in order to determine their best strategy. We define a model with two ISPs, each providing direct connectivity to a fixed proportion of the content and competing in terms of price for end users, who select their ISP based on the price per unit of available content. We analyze and compare thanks to game-theoretic tools three different situations: the case of peering between the ISPs, the case where ISPs do not share their traffic (exclusivity arrangements), and the case where they fix a transfer price per unit of volume. The impact on the network neutrality debate is then discussed.
\end{abstract}

\section{Introduction}

The Internet, initially an academic network connecting universities, has moved to a network used for everyday purposes and open to all. The network is now made of competitive and profit-seeking content providers (CPs) and Internet access providers (or Internet Service Providers (ISPs)). One important principle driving the current network is the universal access principle, meaning that all consumers are entitled to reach meaningful content, whatever the technical limitations of their service [1-3]. But because of large and bandwidth-consuming CPs (for example YouTube), some ISPs to whom those CPs are not directly connected have started to wonder why distant CPs should not be charged by them, with the threat of their traffic not being delivered if they do not accept to pay [4].

Our goal in this paper is to propose a model that will be solved by gametheoretic tools to better understand the relations between the three sets of players that are end users, ISPs and CPs, and to investigate from an economical point of view the relevance of a threat to not transfer the traffic coming from 
competitive ISPs (i.e., coming from CPs not directly connected to the considered ISP). We aim at comparing the three following situations: (i) there is a peering agreement between ISPs who deliver the traffic coming from the competitive ISP at no cost (ii) there is no agreement and no traffic transfer from an ISP to another, limiting as a consequence the content offer (and therefore potentially end-user demand) at each ISP (iii) there is a per-unit-of-volume transit price between the ISPs, which can be obtained from a negotiated agreement between the ISPs or determined by a regulator wishing to maximize social or user welfare. This model with the three different possibilities allows us to determine the best peering relationships between ISPs and the relevance of the threat to break the universality principle.

There exists a recent flourishing literature dealing with network neutrality modeling and analysis. Without being exhaustive, some notable works are [4-11]. But the above papers mainly discuss how revenue should be shared among providers (ISPs and CPs) or how neutrality or non-neutrality affect the providers' investment incentives, innovation at the content level, network quality, and user prices. Actually, the originality of our work relies on 1) the modeling of peering or transit traffic pricing between the ISPs, 2) the modeling of the amount of content directly connected to each ISP, and 3) the use of classical discrete choice models to define how users choose their ISP depending on price, reputation, loyalty, and available content. Indeed, to the best of our knowledge there is no game-theoretical study of the peering and transit pricing agreements between ISPs in the network neutrality context, that is, compared with the non-universal connection threat (Item 1 above). Moreover, usually papers are interested in the relation between one or several CPs with one or several ISPs, but very few address the diversity in terms of content and the fact that the more content is accessible from an ISP, the more likely users are to subscribe to this ISP (Item 2 above). For instance, [12] addresses the competition among CPs in terms of quality of service (QoS), but not on content diversity. The works we have noticed in that last direction are $[13,14]$ where two types of CPs (search engine portal and $\mathrm{P} 2 \mathrm{P}$ ) are considered (not a continuum like here), but the demand per content type is considered independent for simplicity sake. Finally, we choose to describe the user preferences using a discrete choice model, as classically done in econometric analysis but to our knowledge not done in network neutrality analysis (Item 3 above). Remark though that this type of model is related to the stickiness model described in [13,15], but with the additional difference that it is used in $[13,15]$ for a pricing per unit of volume, while we consider a subscription (and flat rate) fee, more representative of the current practice.

The remaining of the paper is organized as follows. Section 2 presents the basic assumptions on the model, the players, and the three scenarii we propose to analyze and compare. The user welfare is also formalized, and general formulas provided. Section 3 analyzes the game when there is peering between ISPs (i.e., with a null transit price) and end users have a full access to all CPs. Section 4 on the other hand describes the users repartition and pricing game among ISPs when they do not agree to exchange their traffic. Section 5 presents the analysis 
when there is a transit pricing agreement, the price being determined from a bargaining phase between ISPs, or by a regulator wishing to maximize user or social welfare. Section 6 concludes the paper by discussing the impact of the disconnection threat on the network neutrality debate.

\section{Model}

\subsection{Model basic components and notations}

We consider two ISPs called $A$ and $B$, in competition for end users. Those users are represented as a continuum of total mass assumed to be 1 without loss of generality.

We also assume that we have a continuum of content (here too of mass 1 without loss of generality) that users may be interested to reach. We call $x$ (respectively $1-x$ ) the proportion of content that is directly connected to the Internet through $A$ (respectively $B$ ). In other words, content providers also have to be connected to one of the two access providers $A$ or $B$, and we just count the proportion $x$ of meaningful content attached to $A$. Note that the proportions $x$ and $1-x$ also encompass the popularity of the contents, which weighs the computation of those proportions: $x$ (respectively $1-x$ ) represents the proportion of the total aggregated flow originating from $\mathrm{CPs}$, that originates from a CP connected to ISP $A$ (respectively, $B$ ). Here, we focus on downlink traffic only, since uplink traffic to content providers is limited to requests and is negligible.

In terms of pricing, we denote by $p_{A}$ (respectively $p_{B}$ ) the access price for a user to provider $A$ (respectively $B$ ). This access price is a flat-rate subscription fee, independent of the amount of volume that the user will download. We also assume that there might be a common price per unit of volume $t$ for the traffic transferred between the two ISPs, defining an economic relationship between ISPs: if $t=0$, this forms the classical peering agreement with no fee, while $t>0$ means a transit pricing usually adopted when there is a strong asymmetry. Those two situations will be investigated in the next sections, with also the situation when the link between ISPs is broken, so that there is no possibility for users attached to an ISP to reach the content attached to the other ISP. All those relations are summarized in Figure 1.

\subsection{Users' preferences}

It remains to define how users choose their ISP. We assume that they select at most one, and the model to describe users' choice is a standard discrete choice model heavily used in travel behavior and econometry in general [16]. We more specifically use a logit model that can approximate any random utility model. In a popular form of the logit model, the valuation (level of satisfaction) of a user $q$ for an alternative or choice $i$ is random and given by

$$
V_{q, i}=v_{i}+\kappa_{q, i},
$$




\section{Content Providers}

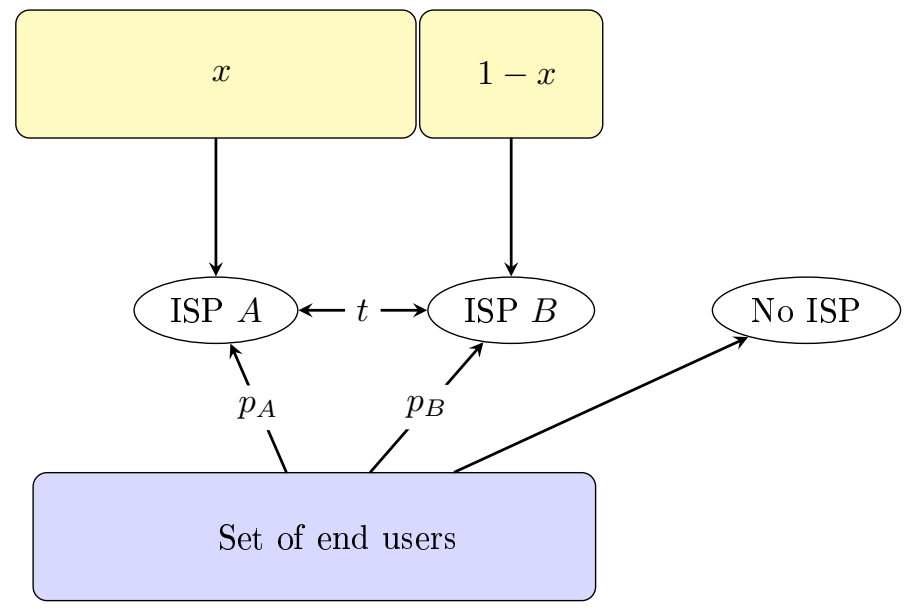

Figure 1: Representation of relations between users, ISPs and CPs

where $v_{i}$ is the average valuation and $\kappa_{q, i}$ a random variable that represents some unobserved random noise. The $\kappa_{q, i}$ are assumed to be independent and to have a Gumbel distribution of mean $0: \mathbb{P}\left[\kappa_{q, i} \leq y\right]=\exp (-\exp (-x / \beta-\gamma))$, where the scale $\beta$ is equal to 1 , and $\gamma$ is Euler constant. In the rest of this paper, since the random variables $\kappa_{q, i}$ (and, as a result, $V_{q, i}$ ) are identically distributed, we will drop the index $q$.

We assume that the average valuation for an ISP $i$ depends on the price per unit of available content $x_{i}>0$ reachable from $i, p_{i} / x_{i}$, through the standard logarithmic relation

$$
v_{i} \stackrel{\text { def }}{=} \alpha \log \left(\frac{x_{i}}{p_{i}}\right),
$$

where $\alpha>0$ is a sensitivity parameter. This type of logarithmic functional has also recently been justified in telecommunications using the context of the Weber-Fechner Law, a key principle in psychophysics describing the general relationship between the magnitude of a physical stimulus and its perceived intensity within the human sensory system [17]. Note that a null price yields an infinite valuation so that a free option will always be preferred to one with charge.

It is important to remark that the mass of available content $x_{A}$ for a user connected through ISP $A$ can differ from the proportion $x$ of CPs directly connected to ISP $A$. This is the case, for instance, if ISPs share their contents: then $x_{A}=x_{B}=1$, all the content is reachable from all ISPs; while if there is no transit between ISPs, $x_{A}=x$ and $x_{B}=1-x$.

We additionally assume that there is an outside option labelled 0 , with average valuation $v_{0}$, representing the possibly negative valuation for not choosing any ISP, and that will be compared with the valuation for ISPs. In accordance 
with (1), we also define $p_{0} \stackrel{\text { def }}{=} \exp \left(-v_{0} / \alpha\right)$, representing the cost associated to not benefitting from any content. In our numerical illustrations, unless specified otherwise, we will take $p_{0}=1$ (or, equivalently, $v_{0}=0$ ).

Each user chooses the option yielding the largest valuation. Following classical discrete choice analysis, a user will choose the option $i \in\{A, B, 0\}$ with probability

$$
\tilde{\sigma}_{i}\left(v_{A}, v_{B}, v_{0}\right) \stackrel{\text { def }}{=} \frac{\exp \left(v_{i}\right)}{\exp \left(v_{A}\right)+\exp \left(v_{B}\right)+\exp \left(v_{0}\right)} .
$$

Consequently, for a given price profile $\left(p_{A}, p_{B}, p_{0}\right)$ and available contents $\left(x_{A}, x_{B}\right)$, that probability is

$$
\sigma_{i}\left(p_{A}, p_{B}, p_{0}\right) \stackrel{\text { def }}{=} \frac{\left(x_{i} / p_{i}\right)^{\alpha}}{\left(x_{A} / p_{A}\right)^{\alpha}+\left(x_{B} / p_{B}\right)^{\alpha}+1 / p_{0}^{\alpha}} .
$$

Several remarks can be made here:

- The probability $\sigma_{i}\left(p_{A}, p_{B}, p_{0}\right)$ is also the proportion of users that will choose option $i \in\{A, B, 0\}$ given that users' choice have been assumed independent and that the total mass of users is 1 .

- The ISPs that set their price to zero will attract all the users. However, their revenue is null in this case.

- The distribution $\left(\sigma_{i}\left(p_{A}, p_{B}, p_{0}\right)\right)_{i \in\{A, B, 0\}}$ uniformly concentrates on choices whose price are minimal when $\alpha$ goes to $\infty$, and is uniform on $\{A, B, 0\}$ when it goes to zero.

- We could assume a different parameter $\alpha$ associated to each option $\alpha_{i}$ for $i \in\{A, B, 0\}$. As a consequence the choice probability turns into

$$
\sigma_{i}\left(p_{A}, p_{B}, p_{0}\right) \stackrel{\text { def }}{=} \frac{\left(x_{i} / p_{i}\right)^{\alpha_{i}}}{\left(x_{A} / p_{A}\right)^{\alpha_{A}}+\left(x_{B} / p_{B}\right)^{\alpha_{B}}+1 / p_{0}^{\alpha_{0}}} .
$$

This heterogeneous case will be discussed for the peering scenario, as well as when ISPs do not exchange any traffic (Sections 3 and 4). We however mainly focus on the homogeneous case for reasons of clarity, and because the paid-transit case in Section 5 cannot be dealt analytically in the heterogeneous case.

- We would also obtain the same result on the choice probability if we remove the $\alpha$ (or $\alpha_{i}$ for the heterogeneous case) from the average valuation, to give $v_{i}=\log \left(\frac{x_{i}}{p_{i}}\right)$, and if we assume that the Gumbel distribution has mean 0 and scale parameter $1 / \alpha$ (or $1 / \alpha_{i}$ for the heterogeneous case, see [18]). In this case, parameter $\alpha$ (or $\alpha_{i}$ ) modifies the variance of the random valuation instead of impacting the mean valuation, the variance being a decreasing function of $\alpha$. Remark though that this other representation would change the expression of the user welfare below, but this will not be investigated for sake of conciseness and to avoid confusion. 


\subsection{ISPs' utilities}

ISPs' utilities are modeled by the revenues they get, that come from the subscription of end users and the transit fees between ISPs, if any. The subscription revenues are proportional to the market share $\sigma_{i}$ of each provider $i$ and its price $p_{i}$. To express the transit fees, recall that $x$ and $1-x$ are the proportions of (weighted by the likeliness of being downloaded) content of ISP $A$ and $B$ respectively, for each customer. Therefore for a customer of ISP $A, x$ can be interpreted as the proportion of the download traffic that will go directly through ISP $A$, while the remaining proportion $1-x$ will have to be delivered through $B$ and then $A$ (if possible), following Figure 1, to reach the user. That then implies some money transfer (transit costs) from ISP $A$ to ISP $B$ in case of transit agreements where the receiver that has asked for the service has to pay for it. The case where the ISP to which content is attached pays to reach the customers (that is, $t<0$ ) could be also considered, and will deserve attention in a future work. However, we only consider for space reason the more relevant situation where providing access to the content is part of the service ISPs offer to users, so that user ISPs bear the possible transfer costs to content.

The total amount of traffic transferred from $B$ to $A$ is $(1-x) \sigma_{A}\left(p_{A}, p_{B}, p_{0}\right)$, paid by $A$ to $B$, while it is $x \sigma_{B}\left(p_{A}, p_{B}, p_{0}\right)$ from $A$ to $B$, paid by $B$. Denote by $\Delta_{A, B}$ the differential amount of traffic that is transferred from ISP $A$ to ISP $B$, i.e.

$$
\Delta_{A, B}=x \sigma_{B}\left(p_{A}, p_{B}, p_{0}\right)-(1-x) \sigma_{A}\left(p_{A}, p_{B}, p_{0}\right) .
$$

Then the respective revenues $U_{A}$ and $U_{B}$ of provider $A$ and $B$ are

$$
\begin{aligned}
& U_{A}\left(p_{A}, p_{B}, p_{0}\right)=\sigma_{A}\left(p_{A}, p_{B}, p_{0}\right) p_{A}+t \Delta_{A, B} \\
& U_{B}\left(p_{A}, p_{B}, p_{0}\right)=\sigma_{B}\left(p_{A}, p_{B}, p_{0}\right) p_{B}-t \Delta_{A, B} .
\end{aligned}
$$

\subsection{User welfare}

The user welfare, or user surplus, is defined as the aggregated net benefit that users get from the system. We consider here as a reference outcome the one with no service, for which the (random) user value is $V_{0}=v_{0}+\kappa_{0}$. From the logit model defined in the previous subsection, the net surplus of a user is what she gains compared to that outside option, i.e., $Z \stackrel{\text { def }}{=} \max \left(0, V_{A}-V_{0}, V_{B}-V_{0}\right)$. Because the total user mass is 1 , the user welfare, that we denote by UW, is $\mathbb{E}[Z]$. Now, remark that for $z \geq 0$,

$$
\begin{aligned}
\mathbb{P}[Z \leq z] & =\mathbb{P}\left[\left(V_{A}-V_{0} \leq z\right) \cap\left(V_{B}-V_{0} \leq z\right)\right] \\
& =\mathbb{P}\left[\left(V_{0} \geq V_{A}-z\right) \cap\left(V_{0} \geq V_{B}-z\right)\right] \\
& =\frac{\exp \left(v_{0}\right)}{\exp \left(v_{0}\right)+\exp (-z)\left(\exp \left(v_{A}\right)+\exp \left(v_{B}\right)\right)} .
\end{aligned}
$$

The last equation is a direct consequence of (2), subtracting $z$ to the average valuations of options $A$ and $B$. 
Therefore, we have

$$
\begin{aligned}
\mathrm{UW}=\mathbb{E}[Z] & =\int_{z=0}^{+\infty} \mathbb{P}[Z>z] d z \\
& =\int_{z=0}^{+\infty} \frac{\exp (-z)\left(\exp \left(v_{A}\right)+\exp \left(v_{B}\right)\right)}{\exp \left(v_{0}\right)+\exp (-z)\left(\exp \left(v_{A}\right)+\exp \left(v_{B}\right)\right)} d z \\
& =\log \left(1+\exp \left(v_{A}-v_{0}\right)+\exp \left(v_{B}-v_{0}\right)\right) \\
& =\log \left(1+\left(x_{A} \frac{p_{0}}{p_{A}}\right)^{\alpha}+\left(x_{B} \frac{p_{0}}{p_{B}}\right)^{\alpha}\right) .
\end{aligned}
$$

As expected, the user welfare is always nonnegative, since users still have the possibility to select no provider as in the reference situation: they only choose to subscribe to a provider if it increases their utility.

In the heterogeneous case, we immediately get from (6)

$$
\mathrm{UW}\left(p_{A}, p_{B}, p_{0}\right)=\log \left(1+p_{0}^{\alpha_{0}}\left(\left(\frac{x_{A}}{p_{A}}\right)^{\alpha_{A}}+\left(\frac{x_{B}}{p_{B}}\right)^{\alpha_{B}}\right)\right) .
$$

\subsection{Scenarios and game analysis}

We recall that in the next sections, we will analyze the pricing game between ISPs (and the transit pricing agreement) in three different scenarios:

1. In the first one, users can access all the content, independently of the chosen ISP, because there is a peering agreement between ISPs ( $p=0$ and $x_{A}=x_{B}=1$ in our model).

2. In the second one, the link between ISPs is broken because they fail to agree on traffic exchange. Therefore, users can only access the content associated with the ISP they have chosen.

3. Finally, in the third scenario, the ISPs define a price per unit of volume they charge each other for the traffic downloaded from the CPs associated to their competitor and transmitted to their customers.

We can notice a hierarchy in the game analysis: at the highest time scale (only for the third scenario), the transit price is chosen (by a bargaining phase or by a regulator). At the intermediate level, ISPs compete on prices to attract customers and maximize their revenue in a classical non-cooperative game. Finally, at the smallest time scale, customers choose their ISP based on available content and price. Remark that those levels are solved by backward induction, anticipating the result at the time scale below.

\section{Scenario 1: Peering between ISPs}

In this scenario, ISPs have a peering agreement, and the users can access all the available content. Consequently $x_{A}=x_{B}=1$ and $p=0$ in our model. For 
given subscription fees $p_{A}$ and $p_{B}$ at providers $A$ and $B$, the proportion of users choosing option $i \in\{A, B, 0\}$ is

$$
\sigma_{i}\left(p_{A}, p_{B}, p_{0}\right)=\frac{\left(1 / p_{i}\right)^{\alpha}}{\left(1 / p_{A}\right)^{\alpha}+\left(1 / p_{B}\right)^{\alpha}+1 / p_{0}^{\alpha}} .
$$

The revenues of $A$ and $B$ can be expressed (with $p=0$ ) as

$$
\begin{aligned}
& U_{A}^{\text {peer }}\left(p_{A}, p_{B}, p_{0}\right)=\sigma_{A}\left(p_{A}, p_{B}, p_{0}\right) p_{A}=p_{A} \frac{\left(1 / p_{A}\right)^{\alpha}}{\left(1 / p_{A}\right)^{\alpha}+\left(1 / p_{B}\right)^{\alpha}+1 / p_{0}^{\alpha}} \\
& U_{B}^{\text {peer }}\left(p_{A}, p_{B}, p_{0}\right)=\sigma_{B}\left(p_{A}, p_{B}, p_{0}\right) p_{B}=p_{B} \frac{\left(1 / p_{B}\right)^{\alpha}}{\left(1 / p_{A}\right)^{\alpha}+\left(1 / p_{B}\right)^{\alpha}+1 / p_{0}^{\alpha}} .
\end{aligned}
$$

Knowing what will be the user repartition for a price profile $\left(p_{A}, p_{B}\right)$ (with $p_{0}$ fixed), ISPs try non-cooperatively to maximize their revenue. The equilibrium notion is that of Nash equilibrium, that would be a price profile from which no ISP can increase its revenue by unilaterally changing its price [19]. Formally, it is a price profile $\left(p_{A}^{\text {peer,NE }}, p_{B}^{\text {peer,NE }}\right)$ such that $\forall p_{A}, p_{B}, U_{A}^{\text {peer }}\left(p_{A}^{\text {peer,NE }}, p_{B}^{\text {peer,NE }}, p_{0}\right) \geq$ $U_{A}\left(p_{A}, p_{B}^{\text {peer,NE }}, p_{0}\right)$ and $U_{B}^{\text {peer }}\left(p_{A}^{\text {peer,NE }}, p_{B}^{\text {peer,NE }}, p_{0}\right) \geq U_{B}\left(p_{A}^{\text {peer,NE }}, p_{B}, p_{0}\right)$.

From the definition, $(0,0)$ is a Nash equilibrium because a unilateral increase in price from an ISP will drive all demand to the other ISP (recall that a free option is always preferred from our discrete choice model, the associated valuation being infinite), hence a revenue still at 0 . Yet, the choice for an ISP to deliver the service for free is a strictly dominated strategy unless the other ISP is doing the same (in which case it is only weakly dominated, since all strategies lead to a null revenue). Consequently, if there is another equilibrium, setting its price to zero cannot be a rational decision for ISPs. In the rest of the paper, we assume that, when another Nash equilibrium exists, ISPs choose strictly positive prices.

The following proposition establishes the existence and uniqueness of a Nash equilibrium in the peering case.

Proposition 1. If $1<\alpha \leq 2$, there exists a unique Nash equilibrium different from (therefore not considered) $(0,0)$, with equilibrium prices

$$
p_{A}^{\text {peer }, N E}=p_{B}^{\text {peer }, N E}=\left(\frac{2-\alpha}{\alpha-1}\right)^{1 / \alpha} p_{0} \stackrel{\text { def }}{=} p^{p e e r, N E} .
$$

The ISPs' revenues are then

$$
U_{A}^{\text {peer }, N E}=U_{B}^{\text {peer }, N E}=\frac{\alpha-1}{\alpha} p^{\text {peer }, N E}=(2-\alpha)^{1 / \alpha} \frac{(\alpha-1)^{1-1 / \alpha}}{\alpha} p_{0} .
$$

The case when $\alpha \leq 1$ results in infinite prices at equilibrium, and $\alpha \geq 2$ leads to a price war, i.e. providers decreasing their prices to 0 , so that $(0,0)$ is the unique equilibrium. 
Proof. We consider the derivatives of ISPs' revenues with respect to their own prices. For $i \in\{A, B\}$, we obtain

$$
\frac{\partial U_{i}^{\text {peer }}}{\partial p_{i}}=\sigma_{i}\left(1-\alpha\left(1-\sigma_{i}\right)\right)
$$

with $\sigma_{i}$ given in (7).

When $\alpha \leq 1$, the derivatives are always strictly positive, and ISPs set infinite prices.

We now consider the case $\alpha>1$. Remark that $\sigma_{i}$ is a strictly decreasing function of $p_{i}$ on $\mathbb{R}_{+}$, going from 1 to 0 , when the competitor sets a strictly positive price. Therefore, from (8), for each value $p_{j}>0$ of its competitor's price, provider $i$ has a unique best-reply price, that is strictly positive and such that $\alpha\left(1-\sigma_{i}\right)=1$, i.e. $\sigma_{i}=1-\frac{1}{\alpha}$. Therefore, when $\alpha>1$ there can be only one Nash equilibrium ( $\left.p_{A}^{\text {peer,NE }}, p_{B}^{\text {peer,NE }}\right)$ with positive prices, that is such that $\sigma_{A}=\sigma_{B}=1-\frac{1}{\alpha}$. We immediately notice that such an equilibrium can only exist if $\alpha \leq 2$, because we always have $\sigma_{A}+\sigma_{B} \leq 1$. In that case, solving $\sigma_{A}=\sigma_{B}=1-\frac{1}{\alpha}$ based on ( 7 ) easily leads to the Nash equilibrium prices provided in the proposition.

To analyze the case when $\alpha>2$, we explicitly express the best-reply price function $\mathrm{BR}_{i}\left(p_{j}\right)$ of provider $i$ when its competitor sets a strictly positive price $p_{j}$. That price is the value $p_{i}$ such that $\sigma_{i}=1-\frac{1}{\alpha}$, i.e., $\operatorname{BR}_{i}\left(p_{j}\right)=p_{i}=$ $\left((\alpha-1)\left(p_{j}^{-\alpha}+p_{0}^{-\alpha}\right)\right)^{-1 / \alpha}$. Remark that when $\alpha \geq 2$, since $p_{0}>0$ we have $(\alpha-1)\left(p_{j}^{-\alpha}+p_{0}^{-\alpha}\right)>p_{j}^{-\alpha}$ and therefore $\mathrm{BR}_{i}\left(p_{j}\right)<p_{j}$. In other terms, when a provider sets a strictly positive price $p_{j}$, the best reply of its competitor is strictly below that price. That situation leads to a price decrease until providers do not make any revenue.

Notice that the result for $\alpha \leq 1$ corresponds to users with low price sensitivity: when a provider $i$ increases its price $p_{i}$, the demand $\sigma_{i}$ decreases slowly, and the product $p_{i} \sigma_{i}$ increases. In economic terms, this corresponds to a price elasticity $E_{i}$ of demand for provider $i$ (when the other providers keeps its price constant) being small in absolute value, i.e.

$$
E_{i} \stackrel{\text { def }}{=} \frac{p_{i}}{\sigma_{i}} \frac{\partial \sigma_{i}}{\partial p_{i}}=-\alpha\left(1-\sigma_{i}\right) \in(-1,0) .
$$

The opposite situation of prices decreasing to 0 when $\alpha \geq 2$, to attract customers of the competitor, is called price war. It comes from a large pricesensitivity of users (here, the value of $\alpha$ ).

In the heterogeneous case with different $\alpha \mathrm{s}$, proceeding similarly for the proof, and using the fact that $\frac{\partial U_{i}^{\text {peer }}}{\partial p_{i}}=\sigma_{i}\left(1-\alpha_{i}\left(1-\sigma_{i}\right)\right)$, it can be shown that, at equilibrium:

- If there is one $i \in\{A, B\}$ such that $\alpha_{i} \leq 1$, then $p_{i}^{\text {peer,NE }}=\infty$, because the utility is increasing, independently of the parameters $\alpha_{j}$ for $j \neq i$. The opponent $j \neq i$ chooses $p_{j}^{\text {peer,NE }}=\infty$ for the same reason if $\alpha_{j} \leq 1$, and 


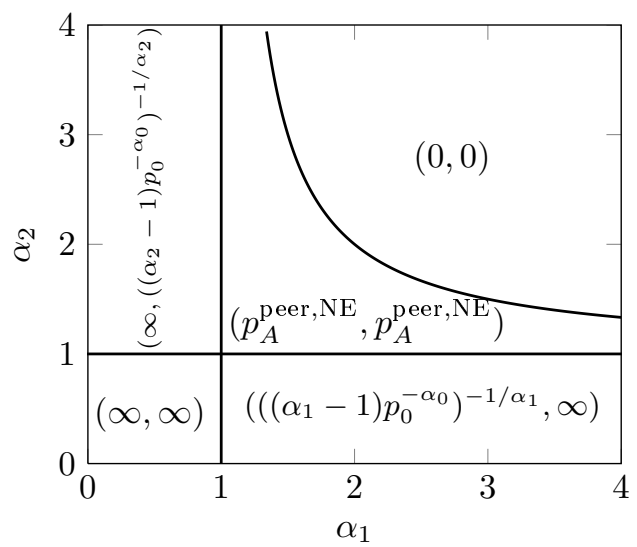

Figure 2: Nash equilibrium in the peering case, in terms of the heterogeneous sensitivies $\left(\alpha_{1}, \alpha_{2}\right)$.

$p_{j}^{\text {peer,NE }}$ such that $\sigma_{j}=1-1 / \alpha_{j}$, i.e., $p_{j}^{\text {peer,NE }}=\left(\left(\alpha_{j}-1\right) p_{0}^{-\alpha_{0}}\right)^{\left(-1 / \alpha_{j}\right)}$ otherwise.

- If $\alpha_{A}, \alpha_{B}>1$,

- If $\left(1 / \alpha_{A}\right)+\left(1 / \alpha_{B}\right) \geq 1$, there is a unique solution to the system of equations with null derivatives, giving

$$
\begin{aligned}
p_{A}^{\text {peer }, \mathrm{NE}} & =\left(\frac{\alpha_{B} \alpha_{A}-\alpha_{B}-\alpha_{A}}{\alpha_{B}\left(1-\alpha_{A}\right)}\right)^{1 / \alpha_{A}} p_{0}^{\alpha_{0} / \alpha_{A}} \\
p_{B}^{\text {peer }, \mathrm{NE}} & =\left(\frac{\alpha_{A} \alpha_{B}-\alpha_{A}-\alpha_{B}}{\alpha_{A}\left(1-\alpha_{B}\right)}\right)^{1 / \alpha_{B}} p_{0}^{\alpha_{0} / \alpha_{B}} .
\end{aligned}
$$

- If $\left(1 / \alpha_{A}\right)+\left(1 / \alpha_{B}\right) \leq 1$, then we again have a price war with, at equilibrium, $p_{A}^{\text {peer,NE }}=p_{B}^{\text {peer, NE }}=0$.

The equilibrium cases in terms of $\left(\alpha_{1}, \alpha_{2}\right)$ are summarized in Figure 2.

Coming back to a unique $\alpha \in(1,2)$, let us analyze the outcome that ISPs can achieve by cooperating, i.e., by setting prices so as to maximize the sum $U_{A}^{\text {peer }}+U_{B}^{\text {peer }}$ of their utilities.

Proposition 2. If $\alpha>1$, the sum of ISPs utilities is maximized by setting prices $p_{A}$ and $p_{B}$ to

$$
p_{\max }^{\text {peer }}=\left(\frac{2}{\alpha-1}\right)^{1 / \alpha} p_{0}
$$

and is equal to $U_{\max }^{\text {peer }}=\frac{\alpha-1}{\alpha} p_{\max }^{\text {peer }}=2^{1 / \alpha} \frac{(\alpha-1)^{1-1 / \alpha}}{\alpha} p_{0}$.

If $\alpha \leq 1$, the optimal pricing is $p_{A}=p_{B}=+\infty$ which results in an infinite $U_{\max }^{\text {peer }}$. 
Proof. First note that if at least one price is zero, then the total revenue of ISPs is null, hence such a price profile cannot be optimal. Thus, either the optimal price of a provider is $\infty$, or it nullifies the derivative.

Remark that the derivatives of $U_{A}^{\text {peer }}+U_{B}^{\text {peer }}$ with respect to $p_{A}$ and $p_{B}$ are

$$
\begin{aligned}
& \frac{\partial\left(U_{A}^{\text {peer }}+U_{B}^{\text {peer }}\right)}{\partial p_{A}}=\frac{\left(p_{A}^{1-\alpha}+\alpha p_{B}^{1-\alpha}-(\alpha-1) p_{A}\left(p_{B}^{-\alpha}+p_{0}^{-\alpha}\right)\right)}{p_{A}^{1+\alpha}\left(p_{A}^{-\alpha}+p_{B}^{-\alpha}+p_{0}^{-\alpha}\right)^{2}} \\
& \frac{\partial\left(U_{A}^{\text {peer }}+U_{B}^{\text {peer }}\right)}{\partial p_{B}}=\frac{\left(p_{B}^{1-\alpha}+\alpha p_{A}^{1-\alpha}-(\alpha-1) p_{B}\left(p_{A}^{-\alpha}+p_{0}^{-\alpha}\right)\right)}{p_{B}^{1+\alpha}\left(p_{A}^{-\alpha}+p_{B}^{-\alpha}+p_{0}^{-\alpha}\right)^{2}} .
\end{aligned}
$$

If $\alpha \leq 1$, those derivatives are always positive, so that the maximum is when $\left(p_{A}, p_{B}\right)=(\infty, \infty)$.

Otherwise, when $\alpha>1$, note that the derivative with respect to $p_{i}(i \in$ $\{A, B\})$ is strictly negative if $p_{i}$ is sufficiently large. The optimal pricing is when the two above derivatives are null, i.e. when

$$
\begin{aligned}
& p_{A}^{1-\alpha}+\alpha p_{B}^{1-\alpha}-(\alpha-1) p_{A}\left(p_{B}^{-\alpha}+p_{0}^{-\alpha}\right)=0 \\
& p_{B}^{1-\alpha}+\alpha p_{A}^{1-\alpha}-(\alpha-1) p_{B}\left(p_{A}^{-\alpha}+p_{0}^{-\alpha}\right)=0 .
\end{aligned}
$$

The difference between those equations leads to $\left(p_{A}-p_{B}\right)\left(p_{A}^{-\alpha}+p_{B}^{-\alpha}+p_{0}^{-\alpha}\right)=0$ which implies $p_{A}=p_{B}$. Then their common value $p_{\max }^{\text {peer }}$ is obtained from any of those equations, yielding the unique solution given in (9).

We now compute the cost of competition for ISPs, through the ratio of their total revenues without cooperation (i.e., at the Nash equilibrium) and with collaboration. That ratio equals

$$
\frac{U_{A}^{\text {peer,NE }}+U_{B}^{\text {peer }, \mathrm{NE}}}{U_{\text {max }}^{\text {peer }}}=2\left(1-\frac{\alpha}{2}\right)^{1 / \alpha} .
$$

Notice that it does not depend on $p_{0}$. That ratio, plotted in Figure 3 in terms of $\alpha$, can also be defined as the inverse of the price of anarchy [20], representing the loss of efficiency in terms of ISPs' revenues due to non cooperation. The figure shows that the larger the user sensitivity to prices, the more ISPs lose by not cooperating.

To further illustrate the equilibrium in terms of $\alpha$, we respectively draw in Figures 4 and 5 the user welfare and total provider revenues (both with and without cooperation) when $1<\alpha<2$. It can be readily checked that a larger sensitivity to price (i.e., a larger $\alpha$ ) yields a larger user welfare and smaller ISPs revenues, and also that a price competition among ISPs is better for users, this improvement increasing with $\alpha$ because the price sensitivity exacerbates competition as highlighted before.

In the heterogeneous case, the derivatives of the sum of providers utilities $U_{A}^{\text {peer }}+U_{B}^{\text {peer }}$ are

$$
\begin{aligned}
\frac{\partial\left(U_{A}^{\text {peer }}+U_{B}^{\text {peer }}\right)}{\partial p_{A}} & =\frac{p_{A}^{1-\alpha_{A}}+\left(1-\alpha_{A}\right) p_{A}\left(p_{B}^{-\alpha_{B}}+p_{0}^{-\alpha_{0}}\right)+\alpha_{A} p_{B}^{1-\alpha_{B}}}{p_{A}^{1+\alpha_{A}}\left(p_{A}^{-\alpha_{A}}+p_{B}^{-\alpha_{B}}+p_{0}^{-\alpha_{0}}\right)^{2}} \\
\frac{\partial\left(U_{A}^{\text {peer }}+U_{B}^{\text {peer }}\right)}{\partial p_{B}} & =\frac{p_{B}^{1-\alpha_{B}}+\left(1-\alpha_{B}\right) p_{B}\left(p_{A}^{-\alpha_{A}}+p_{0}^{-\alpha_{0}}\right)+\alpha_{B} p_{A}^{1-\alpha_{A}}}{p_{B}^{1+\alpha_{B}}\left(p_{A}^{-\alpha_{A}}+p_{B}^{-\alpha_{B}}+p_{0}^{-\alpha_{0}}\right)^{2}} .
\end{aligned}
$$




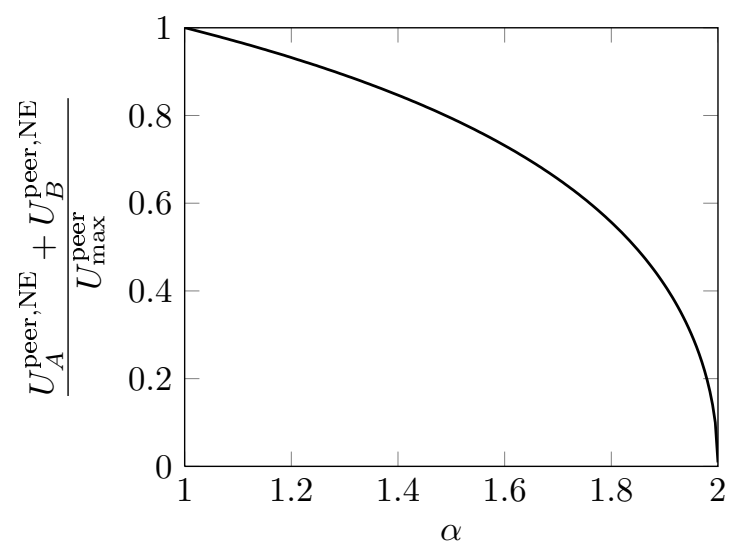

Figure 3: Loss of efficiency due to non-cooperation in terms of $\alpha$ in the peering case

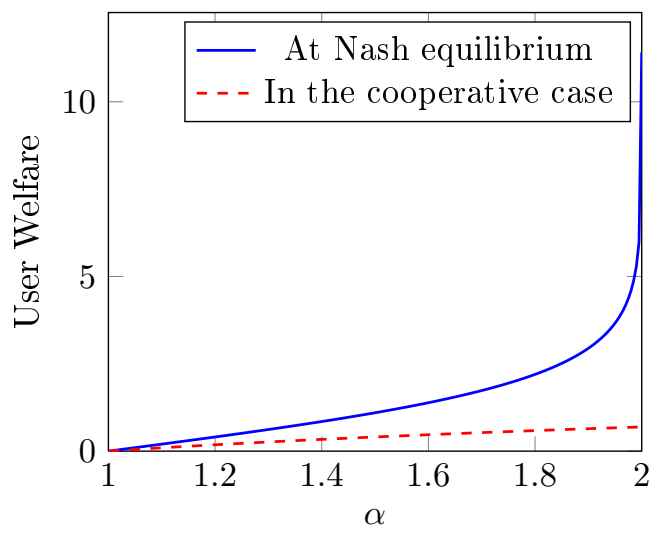

Figure 4: User welfare at Nash equilibrium and in the cooperative case, when peering. 


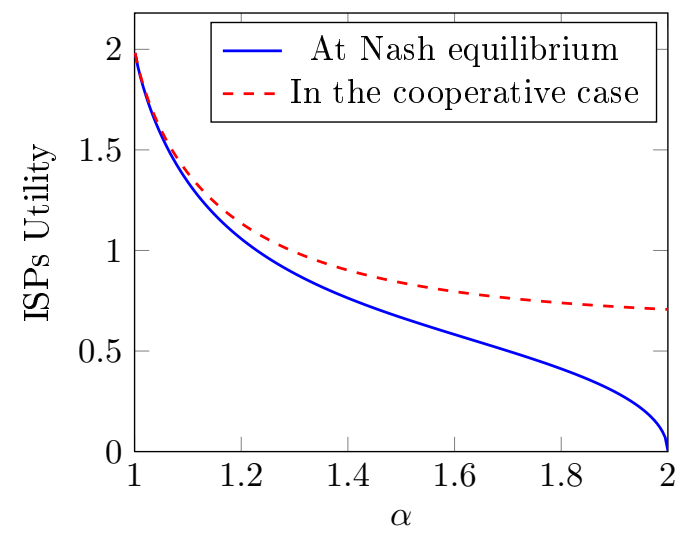

Figure 5: Sum of ISPs' revenues at Nash equilibrium and in the cooperative case, when peering.

Here again, if there is a $i \in\{A, B\}$ such that $\alpha_{i} \leq 1$, the derivative with respect to $p_{i}$ is always positive, and setting $p_{i}=\infty$ is the optimal strategy. Then the total revenue is infinite whatever the value of $p_{j}$ for $j \neq i$. Now, if $\alpha_{A}, \alpha_{B}>1$, the system

$$
\begin{aligned}
& p_{A}^{1-\alpha_{A}}+\alpha_{A} p_{B}^{1-\alpha_{B}}-\left(\alpha_{A}-1\right) p_{A}\left(p_{B}^{-\alpha_{B}}+p_{0}^{-\alpha_{0}}\right)=0 \\
& p_{B}^{1-\alpha_{B}}+\alpha_{B} p_{A}^{1-\alpha_{A}}-\left(\alpha_{B}-1\right) p_{B}\left(p_{A}^{-\alpha_{A}}+p_{0}^{-\alpha_{0}}\right)=0
\end{aligned}
$$

leads to $p_{B}=\frac{\alpha_{B}\left(\alpha_{A}-1\right)}{\alpha_{A}\left(\alpha_{B}-1\right)} p_{A}$. Indeed, $\alpha_{B}$ times the first equation minus $\alpha_{A}$ times the second one gives $\left(\alpha_{B}\left(\alpha_{A}-1\right) p_{A}-\alpha_{A}\left(\alpha_{B}-1\right) p_{B}\right)\left(p_{A}^{-\alpha_{A}}+p_{B}^{-\alpha_{B}}+\right.$ $\left.p_{0}^{-\alpha_{0}}\right)=0$. However, we did not reach an analytical expression for the optimal value of $p_{A}$, that can be computed numerically.

\section{Scenario 2: No traffic exchanged between ISPs}

Because of the potential traffic asymmetry between ISPs, the peering agreement of previous section may not seem satisfactory for one ISP. Two alternatives are considered in this paper: to break the connection between ISPs so that subscribers of a provider have access to the content of that provider only, or to set a transit price such that an ISP has to pay for the content hosted by the competitor and accessed by its own customers (this last scheme is the topic of the next section). Remark here that a threat to break the connection can be raised by both parties: the ISP wishing to deviate from the peering agreement to force its opponent to follow the transaction, but also the ISP to which the deviation is proposed, if the outcome of the threat is not favorable to the proposer. We aim in this section at studying what happens if there is no traffic exchanged between ISPs, and if we can indentify a loser and/or a winner. Remark that 
breaking the transit possibility is not necessarily beneficial for a provider since users have access to less content and may therefore prefer not to subscribe to any provider. It is interesting to note that breaking the connection between ISPs has been implemented in the past; this was for instance the case in 2005 during a dispute between the ISPs Cogent and Level 3, with as a consequence undelivered emails and unreachable web sites for some customers [21].

From our model, if the communication link between ISPs is cut, then $x_{A}=x$ and $x_{B}=1-x$, and from (3), the ISP revenues are

$$
\begin{aligned}
U_{A}^{\text {cut }}\left(p_{A}, p_{B}, p_{0}\right) & =p_{A} \frac{\left(x / p_{A}\right)^{\alpha}}{\left(x / p_{A}\right)^{\alpha}+\left((1-x) / p_{B}\right)^{\alpha}+1 / p_{0}^{\alpha}} \\
U_{B}^{\text {cut }}\left(p_{A}, p_{B}, p_{0}\right) & =p_{B} \frac{\left((1-x) / p_{B}\right)^{\alpha}}{\left(x / p_{A}\right)^{\alpha}+\left((1-x) / p_{B}\right)^{\alpha}+1 / p_{0}^{\alpha}}
\end{aligned}
$$

The next proposition characterizes the outcome of the competition between ISPs in this case. Here too, $\left(p_{A}, p_{B}\right)=(0,0)$ is a Nash equilibrium but the free strategy is a strictly dominated strategy unless the other ISP is doing the same so that we will again ignore this strategy when a Nash equilibrium different from $(0,0)$ exists.

Proposition 3. If $1<\alpha \leq 2$ and $0<x<1$, there exists a unique Nash equilibrium different from $(0,0)$, with equilibrium prices $p_{A}^{\text {cut }, N E}=x p^{\text {cut }, N E}$ and $p_{B}^{c u t, N E}=(1-x) p^{c u t, N E}$, where

$$
p^{c u t, N E}=p^{p e e r, N E}=\left(\frac{2-\alpha}{\alpha-1}\right)^{1 / \alpha} p_{0} .
$$

The ISPs' revenues are then $U_{A}^{c u t, N E}=x U^{c u t, N E}$ and $U_{B}^{c u t, N E}=(1-x) U^{c u t, N E}$, where

$$
U^{c u t, N E}=\frac{\alpha-1}{\alpha} p^{c u t, N E}=(2-\alpha)^{1 / \alpha} \frac{(\alpha-1)^{1-1 / \alpha}}{\alpha} p_{0} .
$$

The case $\alpha<1$ results in infinite prices at equilibrium, and $\alpha \geq 2$ leads to a price war, so that $(0,0)$ is the unique equilibrium.

Proof. The proof mimics the one of Proposition 1, since we again have for each provider $i$

$$
\frac{\partial U_{i}^{\text {cut }}}{\partial p_{i}}\left(p_{A}, p_{B}, p_{0}\right)=\sigma_{i}\left(1-\alpha\left(1-\sigma_{i}\right)\right)
$$

with $\sigma_{i}=\frac{\left(x_{i} / p_{i}\right)^{\alpha}}{\left(x / p_{A}\right)^{\alpha}+\left((1-x) / p_{B}\right)^{\alpha}+1 / p_{0}^{\alpha}}$.

Therefore the best response of ISP $i$ with $j \neq i$ is $\operatorname{BR}_{i}\left(p_{j}\right)=+\infty$ if $\alpha \leq 1$, and

$$
\mathrm{BR}_{i}\left(p_{j}\right)=x_{i}\left((\alpha-1)\left(\left(\frac{x_{j}}{p_{j}}\right)^{\alpha}+\left(\frac{1}{p_{0}}\right)^{\alpha}\right)\right)^{-1 / \alpha}
$$

if $\alpha>1$. In that latter case, an equilibrium with positive prices would correspond to $\sigma_{A}=\sigma_{B}=1-1 / \alpha$, which is only possible if $\alpha \leq 2$, and yields the prices in the proposition. Finally, if $\alpha>2$ we remark that $\mathrm{BR}_{i}\left(p_{j}\right)<p_{j} \frac{x_{i}}{x_{j}}$, so that successive best responses lead to null prices. 
At a Nash equilibrium, ISPs' price (resp. revenue) is equal to the price (resp. revenue) they set (resp. get) in the peering scenario of Section 3, multiplied by the proportion of content they control. As an important conclusion, no ISP $i$ has an interest in breaking the connection, whatever its content share $x_{i}$, because its revenue will be reduced (or the same if $x_{i}=1$ ).

Remark also that the sum of the ISPs' revenue is $x U^{\text {cut,NE }}+(1-x) U^{\text {cut,NE }}=$ $U^{\text {cut }, \mathrm{NE}}=U_{A}^{\text {peer,NE }}=U_{B}^{\text {peer,NE}}$, i.e., exactly the revenue a single provider ( $A$ or $B$ ) would get in case of peering. Surprisingly, if one ISP controls the whole set of contents (for example if $x=1$, i.e., all CPs are attached to ISP $A$ ), then it gets exactly the same revenue as in the first scenario. At a first guess, though, we could have expected that since the opponent has no content, all the customers it has in case of peering would join the monopoly when the transit link is cut, resulting in a monopoly revenue being the sum of revenues in case of peering. But this actually neglects the fact that with the logit model, that encompasses other effects such as reputation, some of the customers of content-less provider prefer not to join any provider (the outside option) than to join the monopoly. In other words, the probability of the outside option when there is a single provider is larger to when there are several, as could be observed in (7). Actually, the Nash equilibrium $\left(p_{A}^{\text {cut,NE }}, p_{B}^{\text {cut,NE }}\right)$ when $0<x<1$ does not converge to the optimal pricing when $x=1$. Indeed, the latter corresponds to a monopoly, for which the optimal price is $p_{\max }^{\operatorname{mono}}=\left(\frac{1}{\alpha-1}\right)^{1 / \alpha}$, whereas $p_{A}^{\text {cut,NE }} \rightarrow\left(\frac{2-\alpha}{\alpha-1}\right)^{1 / \alpha}$ when $x \rightarrow 1$. This shows that, even if an ISP owns a very small proportion of content, its impact is non negligible on the other ISP.

In the previous section we have studied the case where ISPs were cooperating (and peering) in order to maximize the sum their revenues. Though we have not looked at how they split the total revenue among themselves. A question we would like to answer now is: is there a situation such that they both have an interest in cooperating for a given repartition of the total revenue with respect to a price competition and no traffic exchange? If the answer is positive, what is the interval such that the bargaining is satisfying?

Let us consider a revenue sharing agreement among ISPs, when cooperating and peering, such that provider $A$ gets a proportion $\pi_{A}$ of the total revenue $U_{\max }^{\text {peer }}$, and provider $B$ obtains the rest.

ISP $A$ would then prefer that agreement over a pure competitive situation with no traffic exchange, if $\pi_{A} U_{\max }^{\text {peer }} \geq U_{A}^{\text {cut }, \mathrm{NE}}=x U^{\text {cut }, \mathrm{NE}}$. From Propositions 2 and 3 , we obtain that this holds if and only if $\pi_{A} \geq x(1-\alpha / 2)^{1 / \alpha}$. Similarly, such an agreement is acceptable for ISP $B$ if and only if $\pi_{B}=1-\pi_{A} \geq$ $(1-x)(1-\alpha / 2)^{1 / \alpha}$, so that the agreement is stable when

$$
x(1-\alpha / 2)^{1 / \alpha} \leq \pi_{A} \leq 1-(1-x)(1-\alpha / 2)^{1 / \alpha} .
$$

In particular, we remark that $\pi_{A}=x$ is always an acceptable sharing, and that the width of the stable area decreases with $\alpha \in(1,2)$. For example, when $\alpha$ tends to 2 , any revenue sharing of cooperatively obtained revenue is acceptable by ISPs, because competition with such price-sensitive users would lead to a 
price war and null revenues. On the other opposite, remark that when $\alpha$ tends to 1 then only symmetric sharing $\left(\pi_{A}=\pi_{B}=1 / 2\right)$ is acceptable, since in that case each provider could obtain a revenue $U_{\max }^{\text {peer }} / 2$ in the competitive situation with no traffic exchanges. Figure 6 displays that acceptable region for $\pi_{A}$ in terms of $x$, with $\alpha=1.5$.

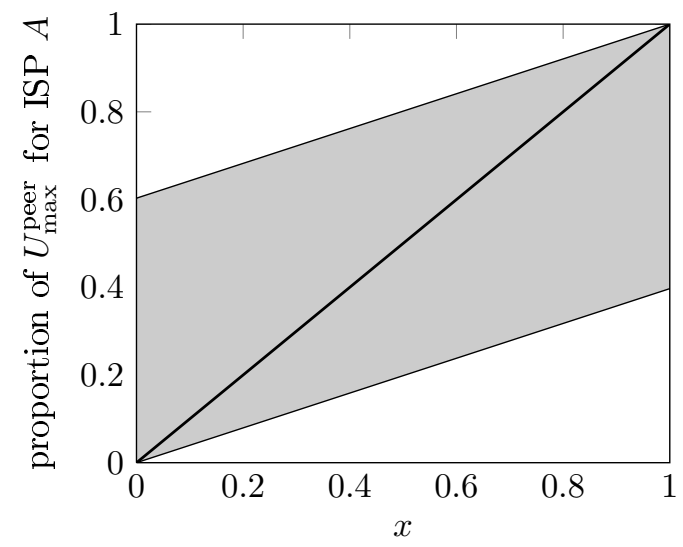

Figure 6: In gray, the set of proportions of $U_{\max }^{\text {peer }}$ that ISP $A$ can receive so that both ISPs accept to cooperate with respect to a broken connection threat. Here $\alpha=1.5$. The line $y=x$ is drawn.

Finally, Figure 7 shows the utilities of ISPs and user welfare as a function of the parameter $\alpha$. The shape of the curves is the same as in Scenario 1, even if the utilities of ISPs are about two times smaller.

In the heterogeneous case with different $\alpha$ s, the results can again be obtained similarly, using that $\frac{\partial U_{i}^{\text {cut }}}{\partial p_{i}}=\sigma_{i}\left(1-\alpha_{i}\left(1-\sigma_{i}\right)\right)$. It can be shown that, at equilibrium:

- If there is one $i \in\{A, B\}$ such that $\alpha_{i} \leq 1$, then again $p_{i}^{\text {cut,NE }}=\infty$. The opponent $j \neq i$ chooses $p_{j}^{\text {cut,NE }}=\infty$ for the same reason if $\alpha_{j} \leq 1$, and $p_{j}^{\text {peer,NE }}=x_{j}\left(\left(\alpha_{j}-1\right) p_{0}^{-\alpha_{0}}\right)^{\left(-1 / \alpha_{j}\right)}$ otherwise.

- If $\alpha_{A}, \alpha_{B}>1$,

- If $\left(1 / \alpha_{A}\right)+\left(1 / \alpha_{B}\right) \geq 1$, there is a unique solution to the system of equations with null derivatives, giving

$$
\begin{aligned}
p_{A}^{\mathrm{cut}, \mathrm{NE}} & =x\left(\frac{\alpha_{B} \alpha_{A}-\alpha_{B}-\alpha_{A}}{\alpha_{B}\left(1-\alpha_{A}\right)}\right)^{1 / \alpha_{A}} p_{0}^{\alpha_{0} / \alpha_{A}} \\
p_{B}^{\mathrm{cut}, \mathrm{NE}} & =(1-x)\left(\frac{\alpha_{A} \alpha_{B}-\alpha_{A}-\alpha_{B}}{\alpha_{A}\left(1-\alpha_{B}\right)}\right)^{1 / \alpha_{B}} p_{0}^{\alpha_{0} / \alpha_{B}} .
\end{aligned}
$$

- If $\left(1 / \alpha_{A}\right)+\left(1 / \alpha_{B}\right) \leq 1$, then we again have a price war with, at equilibrium, $p_{A}^{\text {cut,NE }}=p_{B}^{\text {cut, } \mathrm{NE}}=0$. 


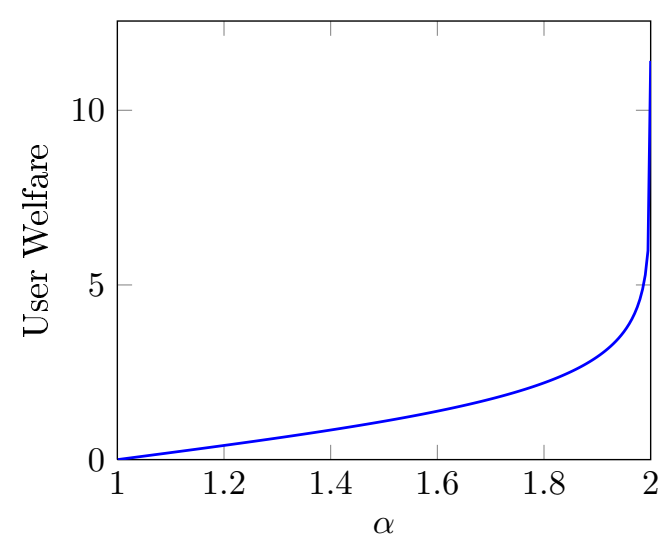

(a) User welfare

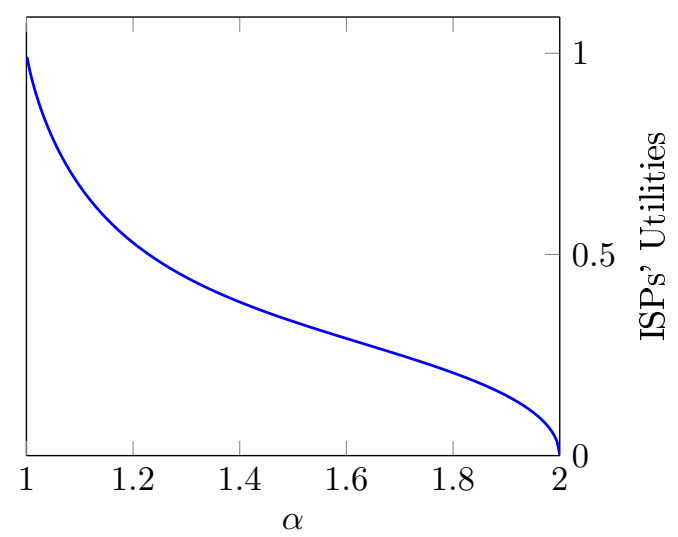

(b) Sum of ISP's utilities

Figure 7: Utilities at Nash equilibrium, with no exchanged traffic, with $x=0.8$.

\section{$5 \quad$ Scenario 3: transit pricing}

In the two previous sections, we have analyzed the competition between ISPs with peering and without any exchanged traffic. We now address the case where the traffic transferred from an ISP to the other is compensated for by some payment. We first determine if a Nash equilibrium exists in that context when such a transit unit price $t>0$ is determined, and we characterize it. Then, we discuss how the transit price can be determined on top of the pricing game for customers, by a regulator or through a bargaining phase between ISPs. Remark that, here again, the transit price is chosen first, but by backward induction, anticipating the equilibrium of the (pricing) game played afterwards.

We begin by studying the game given by the revenue functions (5), with a fixed transit price $t>0$, the case $t=0$ corresponding to our Scenario 1. A first difference with the previous scenarios is that the pricing $(0,0)$ is no longer a Nash equilibrium. Indeed, when an ISP sets its price to zero, it gets no revenue from the users, but only from the transferred traffic charged to the other ISP, while it has to pay for its client accessing the content attached to the competitor ISP. Then two cases appear:

- If the amount of traffic transferred between each ISP is exactly the same, i.e., $\Delta_{A, B}=0$ (see (4)), then both ISPs earn zero revenue. But since at least one ISP has some content associated to it - say, w.l.o.g. ISP $A$, i.e. $x>0$-, that one could get a strictly positive revenue by setting any strictly positive price $p_{A}>0$ : the market shares would be $\theta_{A}=0$ and $\theta_{B}=1$, leading to a revenue $U_{A}=t x>0$.

- If $\Delta_{A, B} \neq 0$, then one ISP (ISP $A$ if $\Delta_{A, B}<0$, ISP $B$ otherwise) gets a strictly negative revenue, whereas it can ensure a nonnegative one by 
setting a strictly positive price and having no subscribers, hence only collecting revenue from traffic transit.

In order to determine the Nash equilibrium of the game, we first analyze the best-response functions of each ISP. The derivatives of ISPs' revenue functions are:

$$
\begin{aligned}
\frac{\partial U_{A}}{\partial p_{A}} & =\frac{K\left(p_{B}\right) p_{A}^{\alpha}+L_{A}\left(p_{B}\right) p_{A}^{\alpha-1}+1}{p_{A}^{2 \alpha}\left(p_{A}^{-\alpha}+p_{B}^{-\alpha}+p_{0}^{-\alpha}\right)^{2}}, \\
\frac{\partial U_{B}}{\partial p_{B}} & =\frac{K\left(p_{A}\right) p_{B}^{\alpha}+L_{B}\left(p_{A}\right) p_{B}^{\alpha-1}+1}{p_{B}^{2 \alpha}\left(p_{A}^{-\alpha}+p_{B}^{-\alpha}+p_{0}^{-\alpha}\right)^{2}},
\end{aligned}
$$

where $K(z) \stackrel{\text { def }}{=}(1-\alpha)\left(z^{-\alpha}+p_{0}^{-\alpha}\right), L_{A}\left(p_{B}\right) \stackrel{\text { def }}{=} \alpha t\left(p_{B}^{-\alpha}+(1-x) p_{0}^{-\alpha}\right)$, and $L_{B}\left(p_{A}\right) \stackrel{\text { def }}{=} \alpha t\left(p_{A}^{-\alpha}+x p_{0}^{-\alpha}\right)$. Notice that $L_{A} \geq 0, L_{B} \geq 0$. Furthermore $K(z)<0$ if and only if $\alpha>1$, so that $U_{A}$ (resp. $U_{B}$ ) is strictly increasing in $p_{A}$ (resp. $p_{B}$ ) when $\alpha \leq 1$. Therefore, as in the previous scenarios, prices tend to infinity when user sensitivity to price is low.

Proposition 4. If $\alpha \leq 1$, the pricing game where ISPs seek to maximize their revenue functions (5) leads to each ISP setting an infinite price.

When $\alpha>1$, we do not reach an analytical expression of the best-response prices of each ISP. Nevertheless, several useful properties are listed in the next proposition, whose proof is given in Appendix A. For convenience, we denote by $\mathrm{BR}_{A}^{t}\left(p_{B}\right)$ and $\mathrm{BR}_{B}^{t}\left(p_{A}\right)$ the best-response correspondences for, respectively, ISP $A$ and $B$ with transit price $t$.

Lemma 1. Assume $\alpha>1$. For every $x \in[0,1]$, the best-response price correspondence of each ISP to the competitor pricing strategy satisfies the following properties:

(i) it is single-valued,

(ii) it is continuous,

(iii) it is uniformly bounded with strictly positive bounds. Furthermore, each ISP can ensure a strictly positive net revenue (that includes user subscriptions and transit prices).

(iv) it increases with the transit price $t$, i.e. $\forall p_{A}, p_{B}, t, r$,

$$
B R_{A}^{t}\left(p_{B}\right)>B R_{A}^{r}\left(p_{B}\right) \Leftrightarrow B R_{B}^{t}\left(p_{A}\right)>B R_{B}^{r}\left(p_{A}\right) \Leftrightarrow t>r,
$$

(v) $B R_{A}^{t}\left(p_{B}\right)$ is strictly increasing (resp. strictly decreasing, constant) in the competitor's price if $x \frac{\left(\alpha t / p_{0}\right)^{\alpha}}{(\alpha-1)^{\alpha-1}}$ is lower than (resp. greater than, equal to) one. The same result holds for $B R_{B}^{t}\left(p_{A}\right)$ by comparing (1 x) $\frac{\left(\alpha t / p_{0}\right)^{\alpha}}{(\alpha-1)^{\alpha-1}}$ to one. 
Those properties can be used to prove the existence of a Nash equilibrium for the pricing game played among ISPs, in the situation of paid transit.

Proposition 5. Assume that $\alpha>1$. Consider the game where the ISPs compete on their prices $p_{A}$ and $p_{B}$ to maximize their revenue given in (5). Then there exists a Nash equilibrium $\left(p_{A}^{\text {trans }, N E}, p_{B}^{\text {trans }, N E}\right)$ with $p_{A}^{\text {trans }, N E}>0$ and $p_{B}^{\text {trans }, N E}>$ 0 , resulting in strictly positive revenues.

Proof. From Lemma 1, the best-response function of each ISP $i$ is continuous, and bounded by strictly positive values. Let us denote by $m_{i}\left(\operatorname{resp} . M_{i}\right)$ the lower (resp. upper) bound of $\mathrm{BR}_{i}^{t}$. Then consider the application

$$
\begin{aligned}
g:\left[m_{A}, M_{A}\right] \times\left[m_{B}, M_{B}\right] & \mapsto\left[m_{A}, M_{A}\right] \times\left[m_{B}, M_{B}\right] \\
\left(p_{A}, p_{B}\right) & \rightarrow\left(\operatorname{BR}_{A}^{t}\left(p_{B}\right), \mathrm{BR}_{B}^{t}\left(p_{A}\right)\right) .
\end{aligned}
$$

Since $g$ is continuous and $\left[m_{A}, M_{A}\right] \times\left[m_{B}, M_{B}\right]$ is a compact convex subset of $\mathbb{R}^{2}$, from Brouwer's fixed point theorem, it has a fixed point that constitutes a Nash equilibrium with strictly positive prices $\left(p_{A}^{\text {trans,NE }}, p_{B}^{\text {trans,NE }}\right) \in\left[m_{A}, M_{A}\right] \times$ $\left[m_{B}, M_{B}\right]$. The strict positivity of the revenues comes from the fact that, from item (iii) of Lemma 1, each ISP can always ensure a strictly positive revenue, whatever the other ISP price.

Note that, unlike in the previous scenarios, there is no price war when $\alpha \geq 2$ as soon as $t>0$, meaning that both providers benefit from the transit pricing because they then reach a strictly positive revenue. In the following, we show that it is at the expense of the user welfare.

Figure 8 shows a numerical approximation of best-response functions. Since they seem to be convex, this suggests that the Nash equilibrium is unique. However, we did not manage to provide theoretical evidence of that result. With our numerical values $(t=0.1, x=0.8, \alpha=1.5)$, we have $x \frac{\left(\alpha t / p_{0}\right)^{\alpha}}{(\alpha-1)^{\alpha-1}}<1$ and $(1-x) \frac{\left(\alpha t / p_{0}\right)^{\alpha}}{(\alpha-1)^{\alpha-1}}<1$ so that, as the proposition ensures, both best-response functions are increasing.

We know that, for every price transit $t$, there exists a Nash equilibrium. We are now interested in the way the transit price is determined anticipating the fact that ISP will select some Nash equilibrium prices afterwards. The next proposition states that a regulator seeking to optimize the user welfare should impose a null transit pricing.

Proposition 6. Assume that $\alpha>1$. The unit transit price maximizing user welfare is $t=0$, which corresponds to the peering situation between ISPs (Scenario 1).

The proof is given in Appendix B.

A regulator seeking to maximize user welfare will set the transit price to zero. But if the aim is to maximize the ISPs (sum of) utilities, it is no longer the case, as illustrated on Figure 9, where the value of $t$ that maximizes the total 


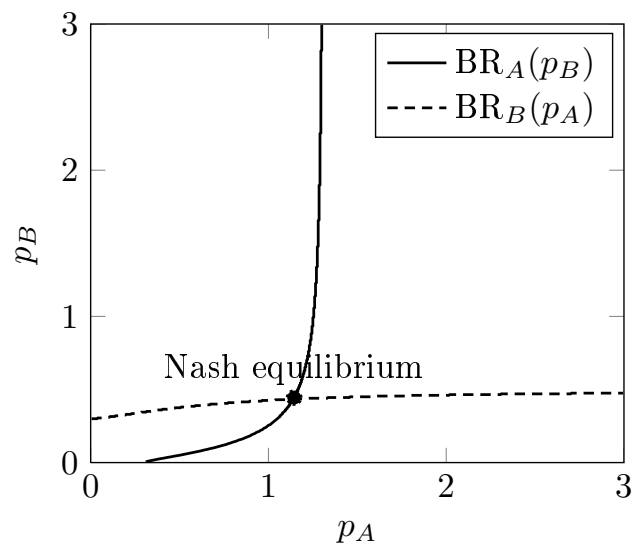

Figure 8: ISP's best response functions, with $x=0.8, \alpha=1.5, p=0.1$.

ISPs' utility is about 0.8. Figure 10 illustrates the evolution of ISPs' utility at the Nash equilibrium when the transit price $t$ varies. For small values of $t$, the utilities of both ISPs increase. One can notice that the utility of the ISP that owns the least content starts to decrease first. We also see that the point that maximizes the sum of utilities is very close to the maximal possible revenue for ISPs if they cooperate, which corresponds to $U_{\max }^{\text {peer }}$ given in the peering scenario. Hence, at this point, the price of anarchy quantifying the loss of ISP revenue due to competition on prices is very close to 1 .

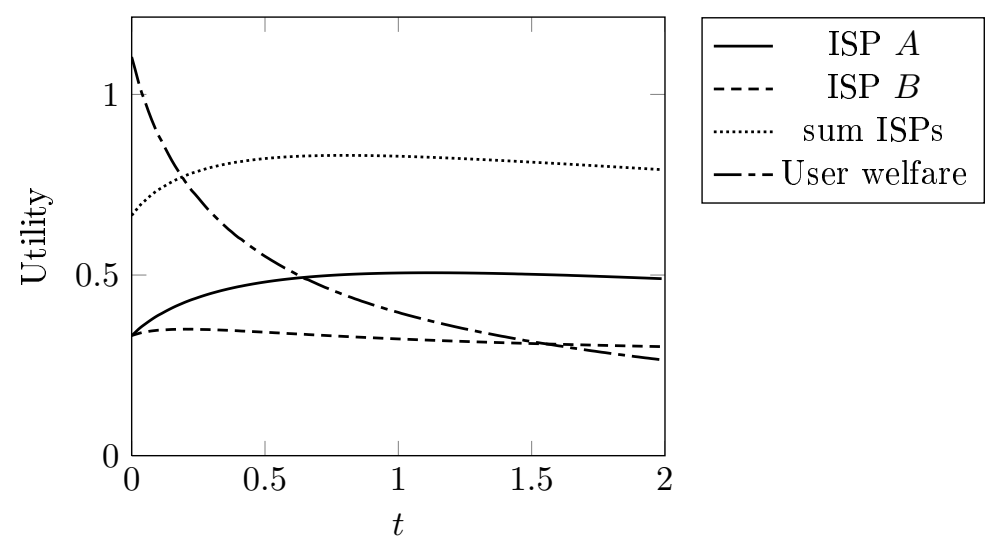

Figure 9: Utilities of the stakeholders at Nash equilibrium prices, as a function of $t$, with $x=0.8, \alpha=1.5$.

We now compare several policies for choosing the transit price. A first policy consists in maximizing the user welfare. From the previous proposition, it corresponds to $t=0$. A second policy consists in maximizing the sum of ISP 


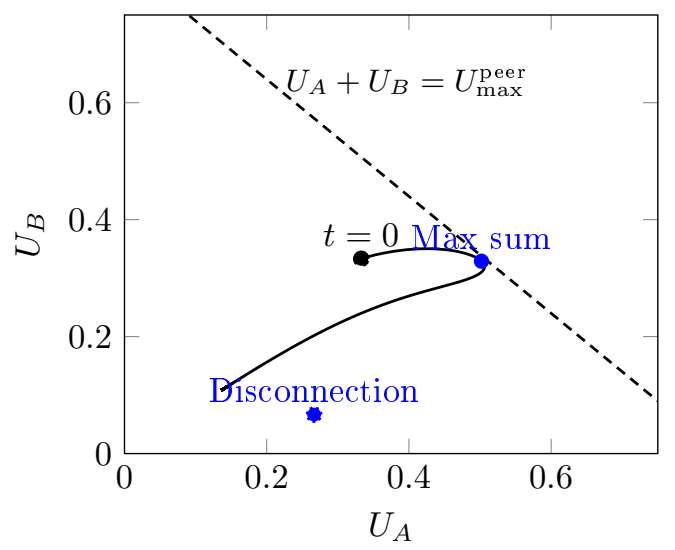

Figure 10: ISP utilities at Nash equilibrium prices, when the transit price $t$ varies. The threat point (i.e., the utilities corresponding to the Nash equilibrium when ISPs are disconnected), the point optimizing the sum of utilities as well as the maximal revenue by cooperating on prices (dashed line) are drawn. Parameter values: $\alpha=1.5, x=0.8$.

utilities (for instance it could be applied by a regulator). And finally, we compare those two policies with the one obtained by a non-cooperative bargaining process between the ISPs.

Here we use the bargaining, or negotiation game proposed in [22]: each ISP independently chooses a set of acceptable transit prices $t$ (prices that ensure a chosen amount of revenue), and if the intersection of those sets is non-empty, the transit price is arbitrarily taken in the intersection, otherwise the threat is executed. This negotiation scheme has several equilibria, but in [22] the most likely to be played is the one maximizing the product of the utilities minus the utility at the threat. In other words, that equilibrium transit price is maximizes

$$
\max \left(0, U_{A}^{\text {trans }, \mathrm{NE}}(t)-U_{A}^{\text {threat }}\right) \cdot \max \left(0, U_{B}^{\text {trans,NE }}(t)-U_{B}^{\text {threat }}\right),
$$

where $U_{i}^{\text {threat }}$ represents the utility that ISP $i$ obtains if the negotiation fails (two cases will be considered in the following).

In Figure 11, we show the impact of taking into account the threat of disconnection during the bargaining process. More precisely, we compare the utility of each ISP obtained with the bargaining procedure when the threat corresponds to the disconnection (i.e., $U_{i}^{\text {threat }}=U_{i}^{\text {cut,NE }}$ ), to the utility achieved when the threat (because of the legislation) is that ISPs are forced to maintain a connection (i.e., $U_{i}^{\text {threat }}=U_{i}^{\text {peer,NE }}$ ). The figure illustrates that the ISP that has the most content (the big ISP) monotonously (in terms of proportion of contents) benefits from the disconnection threat, while the opposite is true for the other ISP (the small ISP). In the case with enforced peering threat, the small ISP still loses some revenue when its weight decreases, but the revenue of the big ISP is no longer monotonous. We observe that the enforced peering threat is a better 
rule than the disconnection threat for the small ISP, while it is the opposite for the big ISP.

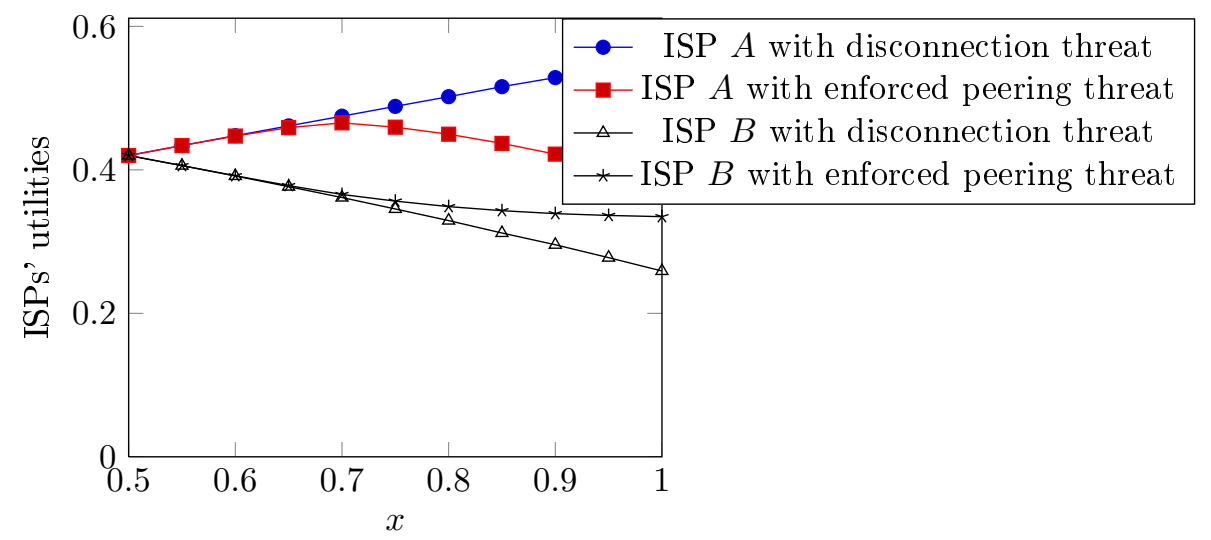

Figure 11: Comparison of each ISP's utility after bargaining process with disconnection and enforced peering threats, when $x$ varies, with $\alpha=1.5$.

In Figure 12, we show how the utilities of ISPs and of the users are impacted by the chosen policy, when the user sensitivity $\alpha$ and the proportion $x$ of content attached to ISP $A$ vary. We first remark that the bargaining with disconnection threat leads to ISP revenues and user welfare very similar to the policy maximizing the sum of ISPs' utilities (the curves of total ISP revenues superimpose). This means that the non-cooperative (bargaining) choice for $t$ with the disconnection threat leads to a nearly optimal point (that takes into account the competition between ISP afterwards) for ISPs. Nevertheless, as one can see, when $x$ is close to 1 or $\alpha$ close to 2 the user welfare is not exactly the same in both cases (see Figure 12 (a) and (c)), hence this is not a general property. One can also observe in Figure 12 (c) and (d) that the utilities are not very sensitive to the repartition of content among ISPs (the parameter $x$ ), except for the bargaining with enforced peering threat. In particular, the solution maximizing user welfare does not depend on $x$, hence utilities are constant in that case. Finally, the bargaining solution obtained with the enforced peering threat is almost optimal for users (resp. ISPs) when their sensitivity $\alpha$ is low (resp. high). The difference of content owned by each ISP also decreases the utility of ISPs in this case, while the achieved utility is optimal when contents are equally shared.

\section{Conclusions}

In this paper, we have studied different transit agreements among ISPs, in a competitive context where users seek for content at a cheap price and traffic may need to be transferred between ISPs. Competition on prices among ISPs is based 


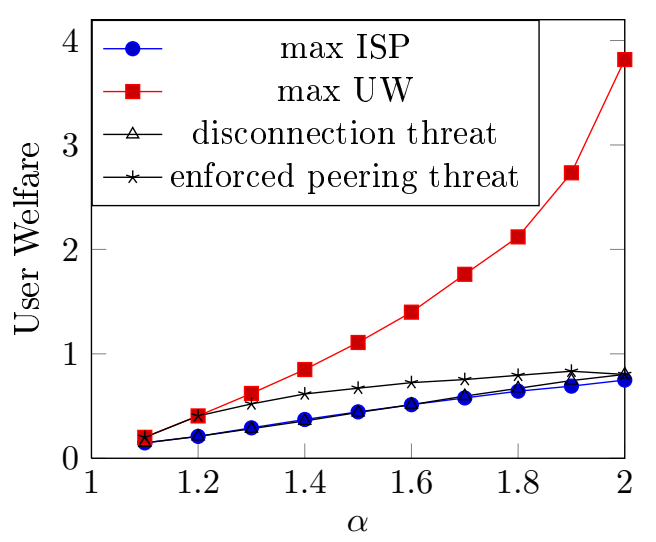

(a) $x=0.8$

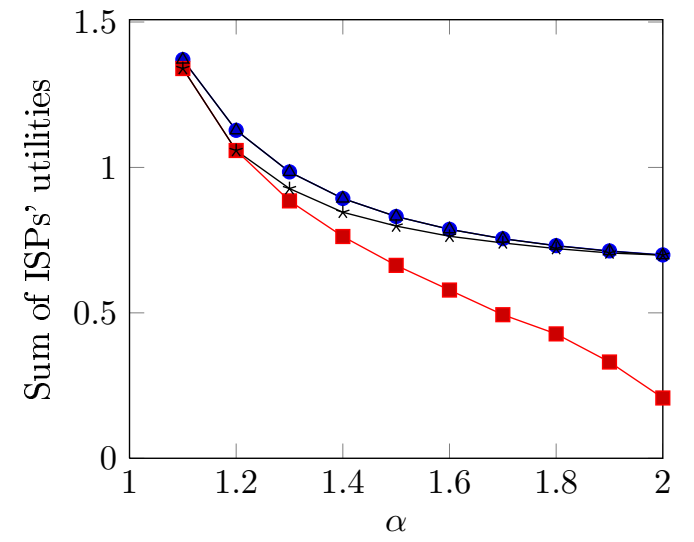

(b) $x=0.8$

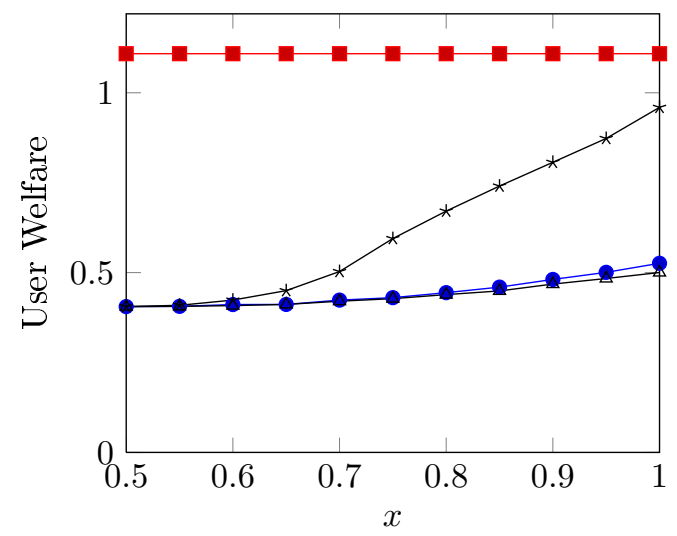

(c) $\alpha=1.5$

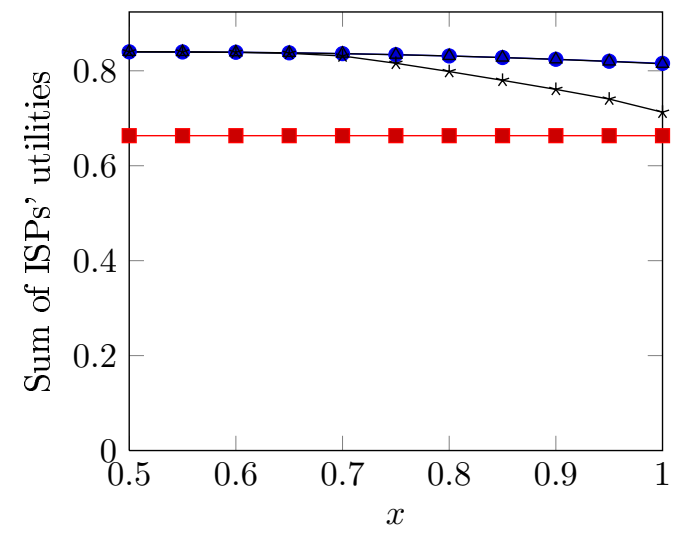

(d) $\alpha=1.5$

Figure 12: ISPs' utilities and user welfare for different policies for choosing $t$, when $\alpha$ and $x$ vary. 
on a user discrete choice model that also encompasses user price sensitivities and some reputation effects, so that user repartition varies continuously with the providers' prices.

For three possible transit agreements (peering, no transit, paid transit), we have analyzed the competition game played among ISPs, and determined the Nash equilibrium prices and revenues, as well as the outcomes when ISPs decide to cooperate. Our results suggest that the scenario where no transit is performed by ISPs should never be chosen, since no stakeholder benefits from it. Surprisingly, we also observed that under price competition, paid transit can be preferred to peering by both ISPs. This especially holds when users are highly sensitive to price: paid transit is then the only agreement under which ISPs can ensure strictly positive revenues, avoiding a price war, for any strictly positive value of the transit unit price. Paid transit therefore appears as the best solution to ensure ISP rentability in a highly competitive context like the current Internet ecosystem, where customers are very volatile and frequently switch providers.

Moreover, the transit price can also be used by a regulatory entity to drive the ISP price to a desirable direction, be it in terms of global ISP revenues (the optimal revenue when ISP collaborate can be approached without collaboration through a proper choice of the transit price) or of user welfare (that can be favored if a sufficiently low transit price is imposed).

Finally, if the transit price is fixed among ISPs through a negotiation, our study suggests that a limited regulation consisting in imposing transit agreements (i.e., imposing that transit be performed to ensure a global connectivity, but at a price chosen by the ISPs) benefits to users, who eventually perceive lower prices, and a higher welfare. Such a regulation indeed reduces the bargaining power of the ISPs controlling the most content, and hence favors the emergence and survivability of new ISPs with less content. Without this regulation, our numerical results suggest that bargaining (with a disconnection threat) leads to an outcome very close to the one where ISPs cooperate to maximize their total revenue.

Coming back to the Network Neutrality debate, and the request from ISPs to be rewarded for transit, we find that our results corroborate their claim and concerns, since null transit prices may lead to ISPs making no revenue despite their infrastructure investments. The results in this paper therefore support the transit price scenario, that is also likely to be chosen by ISPs if the choice is theirs, so that the need for regulation is not obvious. However, this paper also suggests that a limited regulation (enforcing global connectivity) does prevent incumbent ISPs from having a dominant position in the bargaining, and favors competition and users. Given the results presented here, we would advocate that such a minimal regulation be imposed, and that other choices be left to the stakeholders (here, the ISPs), remaining consistent with the freedom spirit that prevailed at the beginnings of the Internet.

As a direction for future work, we would like to investigate the case where the transit ISPs are paid for transferring the external content to their customers, instead of their being charged in this paper. This would correspond to another 
interpretation of the service offered by ISP, where ISPs would sell to content providers the access to their networks, while in this paper they sell to their users the access to all content. That other direction would be worth considering.

\section{Acknowledgment}

This work was supported by Inria's cooperative research action MENEUR (see http://www.irisa.fr/dionysos/pages_perso/tuffin/MENEUR/), Euro-NF Network of Excellence (see http://euronf.enst.fr/) through INNIS project, and the French research agency through the CAPTURES project (see http://captures.inria.fr/).

\section{References}

[1] F. C. Commission, "Order in the matter of preserving the open internet, broadband industry practices," FCC, Tech. Rep., April 2010.

[2] M. Lemley and L. Lessig, "The end of end-to-end: Preserving the architecture of the internet in the broadband era," 48 UCLA Law Review 4, 2001.

[3] S. Wong, R.-M. J., and E. Altman, "Public consultations on Net Neutrality 2010: USA, EU and France," SSRN, Tech. Rep., 2010, http://papers.ssrn. com/sol3/papers.cfm?abstract_id $=1684086$.

[4] T. Lenard and R. E. May, Net Neutrality or Net Neutering: Should Broadband Internet Services be Regulated. Springer Verlag, 2006.

[5] F. Boussion, P. Maillé, and B. Tuffin, "Net neutrality debate: Impact of competition among isps," in Proceedings of the Fourth International Conference on COMmunication Systems and NETworkS (COMSNETS), Bangalore, India, 2012.

[6] R. Ma, D.-M. Chiu, J. Lui, V. Misra, and D. Rubenstein, "Interconnecting eyeballs to content: A Shapley value perspective on isp peering and settlement," in Proceedings of the International Workshop on Economics of Networked Systems (NetEcon), 2008, pp. 61-66.

[7] _ _ , "On cooperative settlement between content, transit and eyeball internet service providers," in Proceedings of the ACM International Conference on Emerging Networking EXperiments and Technologies (CoNEXT), 2008.

[8] J. Musacchio, J. Walrand, and G. Schwartz, "Network neutrality and provider investment incentives," in Signals, Systems and Computers, $200 \%$. ACSSC 2007. Conference Record of the Forty-First Asilomar Conference on, nov. 2007, pp. $1437-1444$.

[9] C. Saavedra, "Bargaining power and the net neutrality debate," 2009, sites. google.com/site/claudiasaavedra/attachments/bargaining_power.pdf. 
[10] E. Altman, P. Bernhard, S. Caron, G. Kesidis, J. Rojas-Mora, and S. Wong, "A study of non-neutral networks with usage-based prices," in Proceedings of the 3rd ETM Workshop, Amsterdam, The Netherlands, September 2010.

[11] E. Altman, A. Legout, and Y. Xu, "Network non-neutrality debate: An economic analysis," in Proceedings of Networking 2011. Barcelona, Spain: LNCS 6641, Springer Verlag, May 2011, pp. 68-81.

[12] P. Njoroge, A. Ozdaglar, N. Stier-Moses, and G. Weintraub, "Investment in two sided markets and the net neutrality debate," Columbia University, Decision, Risk and Operations Working Papers Series, Tech. Rep. DRO2010-05, 2010.

[13] S. Caron, G. Kesidis, and E. Altman, "Application neutrality and a paradox of side payments," in Proceedings of the third International Workshop on Re-Architecting the Internet (ReArch 2010), Philadelphia, USA, November 2010.

[14] G. Kesidis, "Side-payment profitability and interacting eyeball isps under convex demand-response modeling congestion-sensitive applications," arXiv, Tech. Rep. 1110.1779, October 2011.

[15] —_, "Side-payment profitability depending on sensitivity of linear-demand to price of access and content," Pennsylvania State University, Department of Computer Science and Engineering, Tech. Rep. 11-003, 2011.

[16] M. Ben-Akiva and S. Lerman, Discrete choice analysis. The MIT Press, Cambridge, Massachusetts, 1985.

[17] P. Reichl, R. Schatz, and B. Tuffin, "Logarithmic laws in service quality perception : Where microeconomics meets psychophysics and quality of experience," Telecommunication Systems, 2011, to appear, DOI: 10.1007/s11235011-9503-7.

[18] J. Hofbauer and W. Sandholm, "On the global convergence of stochastic fictitious play," Econometrica, vol. 70, no. 6, pp. 2265-2294, 2002.

[19] M. Osborne and A. Rubinstein, A Course in Game theory. MIT Press, 1994.

[20] E. Koutsoupias and C. Papadimitriou, "Worst-case equilibria," in Proc. of 16th Annual Symposium on Theoretical Aspects of Computer Science (STACS 1999), vol. 1563 of Lecture Notes in Computer Science, 1999, pp. 404-413.

[21] S. Cowley. (2005, 06) ISP spat blacks out net connections. InfoWorld. http://www.infoworld.com/t/networking/isp-spat-blacks-outnet-connections-492. 
[22] J. Nash, "Two-person cooperative games," Econometrica: Journal of the Econometric Society, pp. 128-140, 1953.

[23] C. Berge, Espaces topologiques. Fonctions multivoques, ser. Collection Universitaire de Mathématiques. Paris: Dunod, 1959, vol. III.

\section{A Proof of Lemma 1}

Proof. Recall that, by definition, $K(v) \stackrel{\text { def }}{=}(1-\alpha)\left(v^{-\alpha}+p_{0}^{-\alpha}\right)$, and $L_{A}(v) \stackrel{\text { def }}{=}$ $\alpha t\left(v^{-\alpha}+(1-x) p_{0}^{-\alpha}\right)$, so that $K<0$ if $\alpha>1$, and $L_{A} \geq 0$.

We provide here the proof for ISP $A$ only, as it is symmetric for ISP $B$.

(i) The derivative (10) of the revenue function of ISP $A$ is strictly positive for $p_{A}$ small enough, and strictly negative for $p_{A}$ large enough. It follows that a best response must satisfy $\frac{\partial U_{A}}{\partial p_{A}}=0$. We did not reach any closed form solution to this equation, thus we are naturally led to studying the sign of the derivative of $U_{A}$. Notice that the sign and zeros of this derivative are the same as those of the function

$$
S(u, v) \stackrel{\text { def }}{=} K(v) u^{\alpha}+L_{A}(v) u^{\alpha-1}+1,
$$

with $u=p_{A}$ and $v=p_{B}$. Since $\alpha>1, K(v)<0$. Looking at the derivative $\frac{\partial S}{\partial u}$ shows that $S$ is increasing if $u \leq \frac{1-\alpha}{\alpha} \frac{L_{A}(v)}{K(v)}$, and strictly decreasing otherwise. Now, remark that for every $v, S(0, v)>0$ and $\lim _{u \rightarrow \infty} S(u, v)=-\infty$. Since $S$ is continuous, it follows that the equation $S(\cdot, v)=0$ has a unique solution, so that the best-response function is single valued. Let us denote by $\bar{u}(v)$ the unique solution to $S(\cdot, v)=0$, which is also the best-reponse to $p_{B}=v$. One can remark that $S(u, v)$ is positive if and only if $u \leq \bar{u}(v)$. This gives us a criterion to compare a value $u$ with the root $\bar{u}(v)$, that we will frequently use in the rest of the proof.

(ii) The continuity is a consequence of Berge's maximum theorem [23]. The hypotheses of the proposition are valid here, so that the best-response price correspondence is upper hemicontinuous. Since that correspondence is singlevalued, it is a continuous function.

(iii) From the previous analysis, we have for all $v$

$$
\bar{u}(v)>\frac{1-\alpha}{\alpha} \frac{L_{A}(v)}{K(v)}=\frac{t\left(v^{-\alpha}+(1-x) p_{0}^{-\alpha}\right)}{v^{-\alpha}+p_{0}^{-\alpha}} \geq t(1-x),
$$

hence a uniform lower bound for $\bar{u}(v)$ that is strictly positive as soon as $x<1$. In the case $x=1$, our bound goes to zero when $v \rightarrow \infty$. However, we can directly see from (11) that we have $\lim _{v \rightarrow \infty} \bar{u}(v)=\frac{p_{0}}{(\alpha-1)^{1 / \alpha}}$, which is strictly positive. By the continuity of the best-response, there exists $v_{0}>0$ such that

$$
v \geq v_{0} \Rightarrow \bar{u}(v) \geq \frac{p_{0}}{2(\alpha-1)^{1 / \alpha}} \stackrel{\text { def }}{=} C_{1} .
$$


On the other hand, from (12) we have

$$
v \leq v_{0} \Rightarrow \bar{u}(v) \geq \frac{t}{1+p_{0}^{-\alpha} v_{0}^{\alpha}} \stackrel{\text { def }}{=} C_{2},
$$

and therefore $\bar{u}(\cdot)$ is also uniformly bounded on $\mathbb{R}^{+}$by $\min \left(C_{1}, C_{2}\right)$, that is a strictly positive constant when $x=1$.

For the uniform upper bound, we claim that

$$
\bar{u}(v) \leq u_{0} \stackrel{\text { def }}{=} \max \left(1, \frac{\alpha t}{\alpha-1}, \frac{1+\alpha t(1-x) p_{0}^{-\alpha}}{\alpha-1}\right) .
$$

To check that, it suffices to show that $S\left(u_{0}, v\right)<0$ for all $v>0$. We first have

$$
\begin{aligned}
u_{0} \geq \max \left(\frac{\alpha t}{\alpha-1}, \frac{1+\alpha t(1-x) p_{0}^{-\alpha}}{\alpha-1}\right) & \geq \frac{1+\alpha t\left(v^{-\alpha}+(1-x) p_{0}^{-\alpha}\right)}{(\alpha-1)\left(v^{-\alpha}+p_{0}^{-\alpha}\right)} \\
& =\frac{1+L_{A}(v)}{-K(v)},
\end{aligned}
$$

where the second inequality comes from the monotonicity (can be decreasing or increasing) of the right-hand side function in $v$. Hence $-K(v) u_{0}-L_{A}(v) \geq 1$. Since $u_{0} \geq 1$, it follows $u_{0}^{\alpha-1}\left(-K(v) u_{0}-L_{A}(v)\right) \geq 1$, and $S\left(u_{0}, v\right)=K(v) u_{0}^{\alpha}+$ $L_{A}(v) u_{0}^{\alpha-1}+1 \leq 0$.

We claim that playing a best-response always yields a strictly positive revenue for the ISP. Indeed, it can ensure a revenue larger than zero by setting its price to infinity: that way it gets no customers (hence no subscription revenues), and therefore does not pay any transit fees. However it cannot be a best-response, since it should be bounded. Hence the best-response is strictly better, which results in a strictly positive revenue.

(iv) Let $t$ and $r$ be two transit prices, with $t>r$. Assume that the price $v$ of ISP $B$ is fixed, and let $\bar{u}_{r}$ denote the best-response of ISP $A$ to $v$ under transit pricing $r$. Then $(1-\alpha)\left(v^{-\alpha}+p_{0}^{-\alpha}\right) \bar{u}_{r}^{\alpha}+\alpha r\left(v^{-\alpha}+(1-x) p_{0}^{-\alpha}\right) \bar{u}_{r}^{\alpha-1}+1=0$. To show that the best-response under transit pricing $t$ is greater than $\bar{u}_{r}$, it suffices to establish that $(1-\alpha)\left(v^{-\alpha}+p_{0}^{-\alpha}\right) \bar{u}_{r}^{\alpha}+\alpha t\left(v^{-\alpha}+(1-x) p_{0}^{-\alpha}\right) \bar{u}_{r}^{\alpha-1}+1>0$ because, from the proof of $(i), S(u, v)>0$ only if $u<\bar{u}(v)$, and thus $S\left(\bar{u}_{r}, v\right)>0$ means $\bar{u}_{r}<\bar{u}_{t}=\bar{u}(v)$. But $(1-\alpha)\left(v^{-\alpha}+p_{0}^{-\alpha}\right) \bar{u}_{r}^{\alpha}+\alpha t\left(v^{-\alpha}+(1-x) p_{0}^{-\alpha}\right) \bar{u}_{r}^{\alpha-1}+$ $1=\alpha\left(v^{-\alpha}+(1-x) p_{0}^{-\alpha}\right) \bar{u}_{r}^{\alpha-1}(t-r)>0$.

$(v)$ Let $v>w$. We seek to compare $\bar{u}(v)$ and $\bar{u}(w)$. By definition, we have $S(\bar{u}(w), w)=0$. Then, $S(\bar{u}(w), v)=\left(v^{-\alpha}-w^{-\alpha}\right) \bar{u}(w)^{\alpha-1}((1-\alpha) \bar{u}(w)+\alpha t)$. Therefore, $\bar{u}(v)>\bar{u}(w)$ if and only if $(1-\alpha) \bar{u}(w)+\alpha t<0$, i.e. $\bar{u}(w)>$ $\frac{\alpha t}{\alpha-1}$. The last inequality is equivalent to $S\left(\frac{\alpha t}{\alpha-1}, w\right)>0$ which is equivalent to $x \frac{\left(\alpha t / p_{0}\right)^{\alpha}}{(\alpha-1)^{\alpha-1}}<1$. We see that this last inequality does not depend on $w$, which implies that $\bar{u}(v)$ is monotonous. 


\section{B Proof of Proposition 6}

Proof. We prove here that the user welfare (6) at the Nash equilibrium, different from $(0,0)$, with $p=0$ is greater than the user welfare at every Nash equilibrium with $t>0$. Recall that the user welfare is

$$
\mathrm{UW}=\log \left(1+\left(\frac{p_{0}}{p_{A}}\right)^{\alpha}+\left(\frac{p_{0}}{p_{B}}\right)^{\alpha}\right) .
$$

The result follows from the fact that, for each ISP, the price at the Nash equilibrium is the lowest when $t=0$, which we establish below, without loss of generality, for ISP $A$.

Recall that $\mathrm{BR}_{A}^{t}\left(p_{B}\right)$ (resp. $\mathrm{BR}_{B}^{t}\left(p_{A}\right)$ ) is the best-response of ISP $A$ (resp. $B)$ with transit price $t$. We also denote by $\left(p_{A}^{\text {trans, NE }}(t), p_{B}^{\text {trans,NE }}(t)\right)$ the Nash equilibrium with the smallest price for $A$. Then, for all $z<p_{A}^{\text {trans, NE }}(t)$ and every $t>0$ we have

$$
z<\mathrm{BR}_{A}^{t}\left(\mathrm{BR}_{B}^{t}(z)\right)
$$

Indeed, the best-response being lower bounded by a strictly positive value, (13) is verified when $z \rightarrow 0$. If there exists $z<p_{A}^{\text {trans,NE }}(t)$ that does not satisfy the inequality, then by continuity of the best-response functions, there is necessarily $\hat{p}<p_{A}^{\text {trans, NE }}(t)$ for which $\hat{p}=\mathrm{BR}_{A}^{t}\left(\mathrm{BR}_{B}^{t}(\hat{p})\right)$. But this means that $\left(\hat{p}, \mathrm{BR}_{B}^{t}(\hat{p})\right)$ is a Nash equilibrium, which contradicts the hypothesis of $p_{A}^{\text {trans,NE }}(t)$ being the smallest price of ISP $A$ at an equilibrium.

Now, recall from Lemma 1 that the best-response is a strictly increasing function of $t$. Furthermore, while $x \frac{\left(\alpha t / p_{0}\right)^{\alpha}}{(\alpha-1)^{\alpha-1}}<1$, the best-response is a strictly increasing function of the other ISP's price. Let $t$ be small enough so that $\mathrm{BR}_{A}^{t}\left(p_{B}\right)$ is strictly increasing. Let $0<r<t$, and assume that $p_{A}^{\text {trans, NE }}(t)<$ $p_{A}^{\text {trans,NE }}(r)$. Then we have:

$$
\begin{aligned}
p_{A}^{\text {trans }, \mathrm{NE}}(t) & <\mathrm{BR}_{A}^{r}\left(\mathrm{BR}_{B}^{r}\left(p_{A}^{\text {trans }, \mathrm{NE}}(t)\right)\right) \\
& <\mathrm{BR}_{A}^{r}\left(\mathrm{BR}_{B}^{t}\left(p_{A}^{\text {trans }, \mathrm{NE}}(t)\right)\right) \\
& <\mathrm{BR}_{A}^{t}\left(\mathrm{BR}_{B}^{t}\left(p_{A}^{\text {trans }, \mathrm{NE}}(t)\right)\right)=p_{A}^{\text {trans }, \mathrm{NE}}(t) .
\end{aligned}
$$

The first inequality comes from (13) together with the hypothesis $p_{A}^{\text {trans, NE }}(t)<$ $p_{A}^{\text {trans,NE }}(r)$. The second one is due to the fact that $\mathrm{BR}_{B}$ increases with the transit price, and that $\mathrm{BR}_{A}^{r}$ increases with the price set by $B$. The last one is due to the increasingness of $\mathrm{BR}_{A}$ in the transit price, and the last equality stems from $p_{A}^{\text {trans, NE }}(t)$ being a Nash equilibrium price for ISP $A$. Finally, this shows a contradiction. Hence $p_{A}^{\text {trans,NE }}(t)$ increases with $t$ as long as $x \frac{\left(\alpha t / p_{0}\right)^{\alpha}}{(\alpha-1)^{\alpha-1}}<1$.

It remains to show that $p_{A}^{\text {trans, NE }}(t)$ is larger than $p_{A}^{\text {trans,NE }}(0)$ for large values of $t$. We have shown that $p_{A}^{\text {trans, } \mathrm{NE}}(t)$ increases with $t$ while the transit price is below $\hat{t}$ that satisfies $x \frac{\left(\alpha \hat{t} / p_{0}\right)^{\alpha}}{(\alpha-1)^{\alpha-1}}=1$. For that value of $t, \mathrm{BR}_{A}^{\hat{t}}$ is constant, 
and $\mathrm{BR}_{A}^{\hat{t}}=p_{A}^{\text {trans, } \mathrm{NE}}(\hat{t}) \geq p_{A}^{\text {trans, NE }}(0)$. For $t>\hat{t}$, the best-response function is larger than $\mathrm{BR}_{A}^{\hat{t}}$ according to item (iv) of Lemma 1, and therefore larger than $p_{A}^{\text {trans,NE }}(\hat{t})$, independently of $p_{B}$. Hence the Nash equilibrium price of ISP $A$ is larger than $p_{A}^{\text {trans, } \mathrm{NE}}(\hat{t})$ and, finally, than $p_{A}^{\text {trans, NE }}(0)$. 